Case Reports
in Dermatology
Case Rep Dermatol 2020;12:168-173

DOI: 10.1159/000508457

Published online: October 8, 2020
(C) 2020 The Author(s)

Published by S. Karger AG, Basel www.karger.com/cde

This article is licensed under the Creative Commons Attribution-NonCommercial 4.0 International License (CC BY-NC) (http://www.karger.com/Services/OpenAccessLicense). Usage and distribution for commercial purposes requires written permission.

\title{
Fatal Disseminated Mucormycosis in a Hematological Immunocompromised Patient with Extensive Voriconazole Exposure: A Case Report and Review of the Literature
}

\author{
Victoria S. Humphrey ${ }^{a}$ Xiaoxiao Li ${ }^{b}$ Sonal Choudhary ${ }^{b}$ \\ Timothy Patton ${ }^{\mathrm{b}}$ \\ aUniversity of Pittsburgh School of Medicine, Pittsburgh, PA, USA; ${ }^{b}$ Department of \\ Dermatology, University of Pittsburgh Medical Center, Pittsburgh, PA, USA
}

\section{Keywords}

Mucormycosis · Voriconazole $\cdot$ Eschar $\cdot$ Immunocompromised patient

\begin{abstract}
Disseminated mucormycosis is a rare, opportunistic, and aggressive infection typically presenting in immunocompromised patients. Herein, we report a 55 -year-old male with a past medical history of Philadelphia-negative B-cell acute lymphoblastic leukemia who presented with a 2month history of non-painful necrotic ulcers on the nose, knuckles, elbow, foot, and scrotum following 3 months of voriconazole (VRC) exposure in the setting of an unrelated fungal pneumonia. Our case reinforces the virulent and often fatal nature of the disease amongst immunocompromised patients, along with extensive VRC exposure as a possible supplementary risk factor. Disseminated cutaneous mucormycosis should be regarded as a differential diagnosis in all immunocompromised patients, especially those with hematologic malignancies or a history of VRC use, who present with cutaneous ulcerations and eschars.




\section{Case Reports in Dermatology}

\section{Introduction}

Mucormycosis is a rare infection caused by fungi belonging to class zygomycetes, order Mucorales, and can manifest as rhino-orbito-cerebral, cutaneous, pulmonary, gastrointestinal, renal, isolated central nervous system, and disseminated infections [1]. While these organisms are prevalent in the home and environment, as they are common inhabitants of decomposing plant and animal matter, the immune system typically provides a robust defense against infections. However, patients with underlying conditions including severe malnutrition, poorly controlled diabetes mellitus, neutropenia, iron chelation therapy, hematological malignancy, HIV/AIDS, or long-term corticosteroid use can be at higher risk for infection [2]. This report describes a case of disseminated mucormycosis arising in an immunocompromised patient following long-term voriconazole (VRC) use for the treatment of fungal pneumonia.

\section{Case Presentation}

A 55-year-old male with a past medical history of Philadelphia-negative B-cell acute lymphoblastic leukemia status after matched unrelated donor hematopoietic stem cell transplant 86 days before was admitted to the hospital following the development of multiple necrotic ulcers. The ulcers had developed over a period of 2 months after long-term VRC exposure in the setting of an unrelated fungal pneumonia. The patient reported the development of nonpainful necrotizing ulcers on his right 2nd metacarpophalangeal joint a few days prior to a change in therapy from VRC to posaconazole for fungal pneumonia treatment. He developed additional ulcers on his right foot and ankle, bilateral elbows, nose, left foot, and scrotum (Fig. 1). Six days prior to dermatology consult, $40 \mathrm{mg}$ methylprednisolone/day was administered for suspicion of graft-versus-host-disease (GVHD) based on the presence of watery diarrhea, although no skin findings suspicious for cutaneous GVHD were present. The patient was also taking tacrolimus $2.5 \mathrm{mg}$ daily for the prevention of GVHD. At the time of consult, the patient's absolute neutrophil count was $1,600 / \mathrm{mm}^{3}$. Biopsy of the lesion on the right elbow demonstrated ulcer with scale crust, epidermal hyperplasia, and dermal necrosis. Gram and acid-fast bacilli stains were negative. Periodic acid-Schiff and Grocott methenamine stain highlighted ribbon-like branching hyphae in the dermis (Fig. 2). Tissue culture subsequently grew Mucor species. Computed tomography of the chest and maxillofacial showed no evidence of pulmonary or rhino-orbito-cerebral disease.

Initially, the patient was treated with triple antifungal therapy, including amphotericin $b$ $10 \mathrm{mg} / \mathrm{kg}$ daily, caspofungin $50 \mathrm{mg}$ daily, and isavuconazole $372 \mathrm{mg}$ daily. Sargramostim, a recombinant granulocyte monocyte colony-stimulating factor, was initiated, his immunosuppression (tacrolimus and methylprednisolone) was tapered, and plastic surgery, podiatry, and urology were consulted for surgical debridement. Surgical debridement was deferred given his comorbidities, profound pancytopenia, and advanced level of dissemination. The patient's absolute neutrophil count decreased to $500 / \mathrm{mm}^{3}$. Eventually, susceptibilities demonstrated resistance to both isavuconazole and posaconazole; with an amphotericin b susceptibility of 0.06 . Despite all medical efforts, our patient's prognosis continued to deteriorate, and he was discharged and transitioned to home hospice. 


\section{Case Reports in Dermatology}

\section{Discussion}

Disseminated cutaneous mucormycosis is exceedingly rare and deadly, with a mortality rate of $90-100 \%$ [3]. Thus, establishing an early diagnosis is key in the management of this condition. Clinically, cutaneous mucormycosis can present with ulcers and eschars that may be gradual or fulminant in onset, given the angioinvasive nature of the organism. Other presentations can include targetoid lesions, purpuric lesions, tender nodules, and scaly plaques [2]. Blood cultures are usually negative, and cerebrospinal fluid cultures are typically sterile. Radiologically, multiple ( $\geq 10)$ nodules, pleural effusions, and the reverse halo sign are associated with pulmonary disease [4]. A correct diagnosis is primarily achieved via cutaneous biopsy. Polymerase chain reaction-based techniques and matrix-assisted laser desorption ionization-time of flight mass spectrometry have also shown clinical promise [5].

Antifungal therapy, surgical debridement, and minimizing or eliminating predisposing factors for infection are the mainstays of therapy $[6,7]$. Intravenous amphotericin $b$ is for the recommended initial therapy. Early treatment is imperative, as delayed therapy ( $\geq 6$ days following diagnosis) results in an almost twofold mortality at 12 weeks [8]. Posaconazole or isavuconazole can be used as step-down or salvage therapy based upon the patient's tolerance and response. Echinocandins have also demonstrated clinical utility [9].

Aggressive surgical debridement should be performed if the clinical situation allows. Minimization or elimination of predisposing factors, including hyperglycemia, metabolic acidosis, immunosuppressive drugs, deferoxamine administration, and neutropenia is essential to maximizing outcomes. Therapy should continue until clinical resolution is reached. In addition to the above predisposing factors, increases in the incidence of breakthrough mucormycosis have been linked to the widespread administration of VRC in transplant patients and those with hematologic malignancies [10]. After salvage treatment has been exhausted, involvement of palliative care is essential to facilitate goals of care conversations.

In summary, our case reinforces the virulent and often deadly nature of disseminated mucormycosis. Clinicians should have high suspicion, especially in immunocompromised patients with hematologic malignancies and VRC exposure, and a low threshold for biopsy of suspicious cutaneous lesions as early diagnosis and treatment is vital for improving patient outcomes.

\section{Statement of Ethics}

All subjects provided written consent to publish their case (including images). Measures were taken to maintain the privacy of all subjects. This research was conducted ethically in accordance with the World Medical Association Declaration of Helsinki.

\section{Conflict of Interest Statement}

The authors have no conflicts of interest to disclose.

\section{Funding Sources}

The authors did not receive any funding. 


\section{Case Reports in Dermatology}

\section{Author Contributions}

All authors equally contributed to the manuscript, including drafting, revising, and interpretation.

\section{References}

1 Chander J, Kaur J, Attri A, Mohan H. Primary cutaneous zygomycosis from a tertiary care centre in northwest India. Indian J Med Res. 2010 Jun;131:765-70.

2 Castrejón-Pérez AD, Welsh EC, Miranda I, Ocampo-Candiani J, Welsh O. Cutaneous mucormycosis. An Bras Dermatol. 2017 May-Jun;92(3):304-11.

3 Roden MM, Zaoutis TE, Buchanan WL, Knudsen TA, Sarkisova TA, Schaufele RL, et al. Epidemiology and outcome of zygomycosis: a review of 929 reported cases. Clin Infect Dis. 2005 Sep;41(5):634-53.

4 Skiada A, Lass-Floerl C, Klimko N, Ibrahim A, Roilides E, Petrikkos G. Challenges in the diagnosis and treatment of mucormycosis. Med Mycol. 2018 Apr;56(suppl_1):93-101.

5 Ling H, Yuan Z, Shen J, Wang Z, Xu Y. Accuracy of matrix-assisted laser desorption ionization-time of flight mass spectrometry for identification of clinical pathogenic fungi: a meta-analysis. J Clin Microbiol. 2014 Jul;52(7):2573-82.

6 Spellberg B, Walsh TJ, Kontoyiannis DP, Edwards J Jr, Ibrahim AS. Recent advances in the management of mucormycosis: from bench to bedside. Clin Infect Dis. 2009 Jun;48(12):1743-51.

7 Farmakiotis D, Kontoyiannis DP. Mucormycoses. Infect Dis Clin North Am. 2016 Mar;30(1):143-63.

8 Chamilos G, Lewis RE, Kontoyiannis DP. Delaying amphotericin B-based frontline therapy significantly increases mortality among patients with hematologic malignancy who have zygomycosis. Clin Infect Dis. 2008 Aug;47(4):503-9.

9 Ibrahim AS, Bowman JC, Avanessian V, Brown K, Spellberg B, Edwards JE Jr, et al. Caspofungin inhibits Rhizopus oryzae 1,3-beta-D-glucan synthase, lowers burden in brain measured by quantitative PCR, and improves survival at a low but not a high dose during murine disseminated zygomycosis. Antimicrob Agents Chemother. 2005 Feb;49(2):721-7.

10 Trifilio SM, Bennett CL, Yarnold PR, McKoy JM, Parada J, Mehta J, et al. Breakthrough zygomycosis after voriconazole administration among patients with hematologic malignancies who receive hematopoietic stem-cell transplants or intensive chemotherapy. Bone Marrow Transplant. 2007 Apr;39(7):425-9. 


\section{Case Reports in Dermatology}
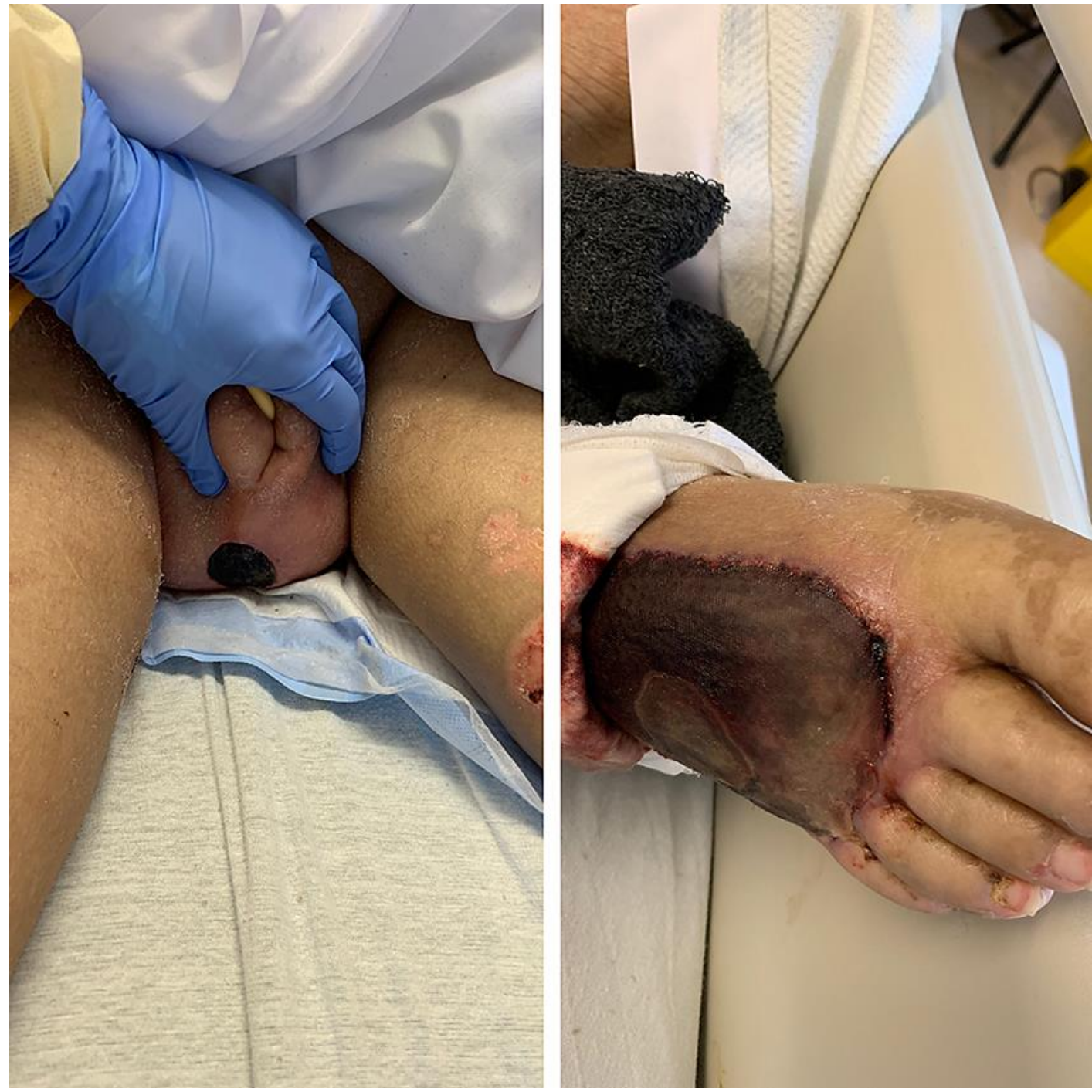

Fig. 1. Scrotum (left panel) and right foot (left panel) with large eschars. 


\section{Case Reports in Dermatology}

\begin{tabular}{l|l}
\hline Case Rep Dermatol 2020;12:168-173 \\
\hline DOI: 10.1159/000508457 & $\begin{array}{l}\text { C 2020 The Author(s). Published by S. Karger AG, Basel } \\
\text { www.karger.com/cde }\end{array}$ \\
\hline
\end{tabular}

Humphrey et al.: Fatal Disseminated Mucormycosis in a Hematological Immunocompromised Patient with Extensive VRC Exposure
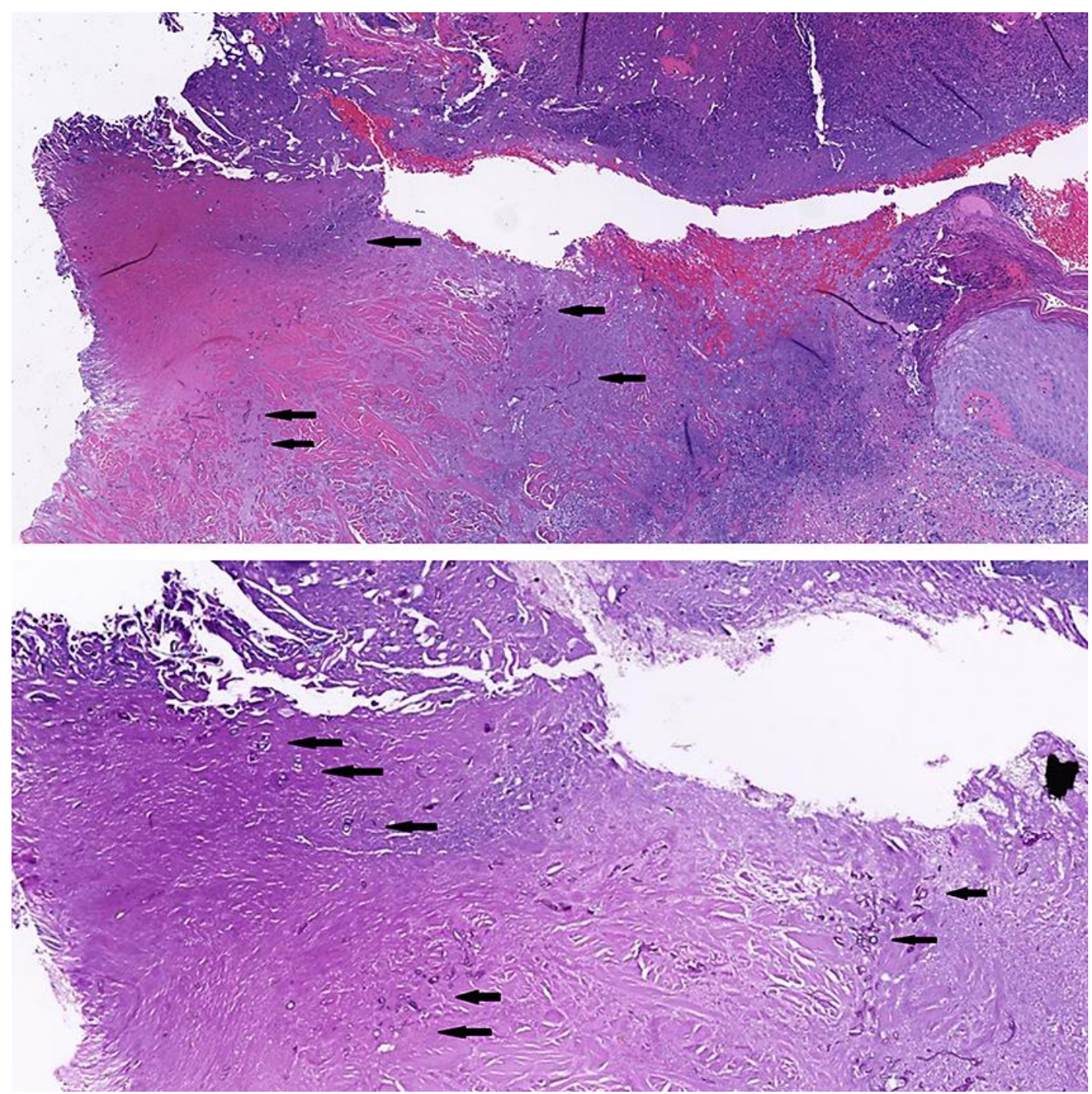

Fig. 2. H\&E (top panel) and Periodic acid-Schiff (bottom panel) highlighting ribbon-like branching hyphae in the dermis. 